\title{
PA-068 CYTOLOGICAL PROFILE OF RED BLOOD CELLS IN HIV-INFECTED PATIENTS: CASE OF THE DOUALA GENERAL HOSPITAL (CAMEROON)
}

Amel Bidias, ${ }^{1}$ Dieudonné Ndjonka, ${ }^{1}$ Clémence Olemba, ${ }^{2}$ Arabo Souleymanou'. 'University of Ngaoundéré, Cameroon; ${ }^{2}$ General Hospital of Douala, Cameroon

\subsection{6/bmjgh-2016-000260.101}

Background Haematological abnormalities have been documented as strong independent predictors of morbidity and mortality in HIV-infected individuals. Those infected with HIV without antiretroviral treatment (ART) have a high prevalence of abnormal blood cells. HIV-1 induced dyserythropoiesis in conjunction with the effects of HIV-related inflammation and/or chronic immune activation. The objective of the study is to identify and characterise the different red cell morphological changes that occur during the evolution of HIV infection in patients according to clinical, biological and therapeutic variables.

Methods A total of 232 patients infected by HIV were included in this cross-sectional and descriptive study conducted at the Douala General Hospital (Cameroon) from June to December 2015. All the patients were screened for red blood cells abnormalities. Blood samples were taken in EDTA tubes for full blood counts (FBC) and blood films. chi-square test was used to compare the variables, and the statistical significance level adopted was p-value under 0.05 .

Results Three quarters of patients in our study had abnormal quantitative or qualitative red blood cells, giving a prevalence of $77.5 \%$. The mean value of haemoglobin was $11.9 \mathrm{~g} / \mathrm{dl}$ with a prevalence of anemia at $61.2 \%$ for all participants. The main red blood cells abnormalities were the anisocytosis (43.1\%), the anisochromia $(34.5 \%)$, the macrocytosis $(24.1 \%)$, the microcytosis $(13.8 \%)$, the hypochromia $(12.9 \%)$ and the poikilocytosis (12.5\%). These abnormalities are statistically significant and are dependent on the severity of the anaemia the WHO clinical stage, the ART duration and the medication regime with all $\mathrm{p}<0.05$.

Conclusions The frequency of cytological abnormalities of red blood cells is high during HIV infection and proportional to the 
severity of the anemia, the duration of antiretroviral therapy diet and its clinical evolution stage. We recommend that reading blood films is systematic of FBC prescription in the monitoring of HIV-infected patients. 